\title{
Guest Editorial SACMAT 2009 and 2010
}

This issue of TISSEC includes extended versions of articles selected from the programs of the 14th and 15th ACM Symposium on Access Control Models and Technologies (SACMAT 2009 and SACMAT 2010), which were held, respectively, in Stresa, Italy on June 3-5, 2009, and in Pittsburgh, USA on June 9-11, 2010. These symposiums continued the SACMAT tradition of being the premier forum for presentation of research results on cutting edge issues of access control, including models, systems, applications, and theory.

SACMAT 2009 received 75 submissions from around the world. After a rigorous review process and program committee discussion, 24 papers were included in the program. SACMAT 2010 attracted a total of 79 papers from Africa, Asia, Australia, Europe and North America. In SACMAT 2010, submissions were anonymous, and each of them was reviewed according to a blind review process by at least three reviewers who are experts in the field. As result of this review process, the SACMAT 2010 program committee selected 19 articles that cover a variety of topics, including RBAC, policy analysis, role engineering and access control in distributed environments.

Based on the original reviews and the feedback from session chairs, two papers from SACMAT 2009 and two papers from SACMAT 2010 were invited for submission for this special issue. The journal submissions went through an additional review process by selected members of the SACMAT 2010 program committee as well as external reviewers. The authors were required to incorporate significant technical extensions into their extended versions. Each article went through two rounds of reviews where the authors were requested to respond to the review comments and update the submissions accordingly. As the result of this review process, we finalized three articles to be included in this special issue. The first two articles are from the SACMAT 2009 program and the third article is from the SACMAT 2010 program.

The first article titled "Group-Centric Secure Information-Sharing Models for Isolated Groups" by Ram Krishnan, Jianwei Niu, Ravi Sandhu, and William H. Winsborough proposes a theory for Group-Centric Secure Information Sharing (g-SIS) with isolated groups and formalizes a family of g-SIS models. The proposed g-SIS approach brings together users and objects in a group from different external sources and facilitates information sharing. The authors focus on the authorization semantics of group operations of join, leave for users and add, remove and create for objects. They use first-order temporal logic to define the core properties and some additional properties related to authorization consequences of these operations. They show that the core properties are logically consistent and mutually dependent. Further, they specify authorization behavior for a family of g-SIS models and prove that these models satisfy the core and selected additional properties.

The second article titled "Combining Discretionary Policy with Mandatory Information Flow in Operating Systems" by Ziqing Mao, Ninghui Li Hong Chen, and Xuxian Jiang proposes an Information Flow Enhanced Discretionary Access Control (IFEDAC) model by combining discretionary policy in DAC with the dynamic information-flow techniques in MAC. The authors describe the design of IFDAC, show its relationship to the existing usable mandatory integrity protection model, and analyze their security properties. The authors also describe their implementations of IFEDAC in Linux and present their evaluation results.

The third article titled "Access Control Policy Translation, Verification, and Minimization within Heterogeneous Data Federations," by Gregory Leighton and Denilson Barbosa deals with the problem of enforcing access control policies across

(c) 2011 ACM 1094-9224/2011/11-ART22 $\$ 10.00$

DOI 10.1145/2043621.2043622 http://doi.acm.org/10.1145/2043621.2043622 
data federations. In particular, the authors investigated the problem of translating existing access control policies to allow the original semantics to be observed while becoming applicable across the entire data federation. The article shows that such a translation is always possible, whereas the verification of translated policies is an intractable problem. As a further contribution, the article presents an algorithmic framework for translating relational access control policies into their XML equivalent expressed in XACML.

We would like to thank all the authors for submitting their research results to this special issue and all the reviewers for their efforts in reviewing the articles and providing valuable feedback to the authors. We are also grateful to Gene Tsudik, Editor-in-Chief, for his guidance and help throughout this process.

—James Joshi
SACMAT 2009 Program Chair
University of Pittsburgh
Barbara Carminati
SACMAT 2010 Program Chair
University of Insubria, Italy
Guest Editors

